\title{
Radiography and tomography system using refractive lenses
}

\author{
C. K. Gary, ${ }^{* 1}$ H. Park, ${ }^{1}$ R.H. Pantell, ${ }^{2}$ Y.I. Dudchik,${ }^{3}$ L. W. Lombardo, ${ }^{1}$ andJ. T. Cremer ${ }^{1}$ \\ ${ }^{1}$ Adelphi Technology, Inc. 981-B Industrial Road, San Carlos, California 94070, USA \\ ${ }^{2}$ Department of Electrical Engineering, Stanford University, Stanford, California 94305, USA \\ ${ }^{3}$ Institute of Applied Physics Problems, Kurchatova 7, Minsk 220064, Belarus
}

\begin{abstract}
A prototype x-ray imaging system was built and tested for high-resolution x-ray radiography and tomography. The instrument consists of a microspot $\mathrm{X}$-ray tube with a multilayer optic, a parabolic compound refractive lens (CRL) made of a plastic containing only hydrogen and carbon, and an x-ray detector. A rotation stage was added for tomography. Images were acquired of both grid meshes and biological materials, and these are compared to images achieved with spherical lenses. We found the best image quality using the multilayer condenser with a parabolic lens, compared to images with a spherical lens and without the multilayer optics. The resolution was measured using a 155 element parabolic CRL and a multilayer condenser with the microspot tube. The experiment demonstrates about $1.1 \mu \mathrm{m}$ resolution.
\end{abstract}

\section{KEYWORDS}

$\mathrm{X}$-ray imaging, tomography, compound refractive lens, high-resolution x-ray imaging, microtomography, small animal imaging.

\section{SYSTEM CONSTRUCTION}

Images were recorded using a Photonic Sciences FDI Imager x-ray camera using a microspot x-ray tube with a multilayer optic and a parabolic compound refractive lens (CRL). The images taken with the parabolic lens microscope are compared to those taken by a bubble $\mathrm{CRL}^{1,2}$ with and without the multilayer optic.

Previously, well-aligned, closely-spaced series of biconcave refractive lenses have been used in a simple microscopes for imaging using synchrotron sources. ${ }^{3,4}$ Each biconcave lens has a focal length of $f^{\prime}=R / \delta$ and the series of $N$ plano-concave unit lenses forms a compound refractive lens (CRL) with a total focal length $f_{0}=f^{\prime} / N$, where the complex refractive index of the unit lens material is $n=1-\delta+i(\lambda \mu / 4 \pi), \delta$ is the index decrement, $\mu$ is the linear attenuation coefficient, $R$ is the radius of curvature of the lens, $\mathrm{t}$ is the thickness of the lens, and $\lambda$ is the $\mathrm{x}$-ray wavelength. ${ }^{5}$

For a thick CRL, the focal length, $F$, is given by ${ }^{6}$

*cgary@adelphitech.com; Phone: 650-598-9800 x 15; Fax: 650-598-9400

Penetrating Radiation Systems and Applications VIII, edited by F. Patrick Doty, H. Bradford Barber, Hans Roehrig,

Proc. of SPIE Vol. 6707, 67070R, (2007) - 0277-786X/07/\$18 · doi: 10.1117/12.735645

Proc. of SPIE Vol. 6707 67070R-1 


$$
\frac{f}{f_{0}}=\frac{\left(\frac{t}{f_{0}}\right)^{1 / 2}}{\sin \left(\frac{t}{f_{0}}\right)^{1 / 2}}
$$

The parabolic CRL is composed of many biconcave parabolic lenses. We melted a plastic containing only carbon and hydrogen and molded it into lenses with Al parabolic shape masters. These low atomic-number materials were chosen for the plastic due to their better x-ray focusing ability compared to higher atomic-number elements. The $\mathrm{Al}$ masters were designed to have a $50-\mu \mathrm{m}$ radius of curvature, and were micromachined. Fig 1 shows a SEM photograph of an Al master. The molded polymer disks, made with the Al master, have a dimple in the center. The polymer in the dimple forms a bi-concave parabolic lens. Those disks are closely stacked inside a stainless tube to form a parabolic CRL.

Such a lens can be used for magnification of an object, behaving like an ordinary optical lens in that it obeys the lens formula, $1 / o+1 / i=1 / f$, where $o$ is the object distance and $i$ is the image distance as shown in Fig. 2. Like a simple optical microscope, the magnification is given by $M=i / o$.

The walls of the lenses absorb x-rays, causing the thicker edges of the biconcave lenses to limit the aperture of the CRL. This absorption aperture as a function of $f$

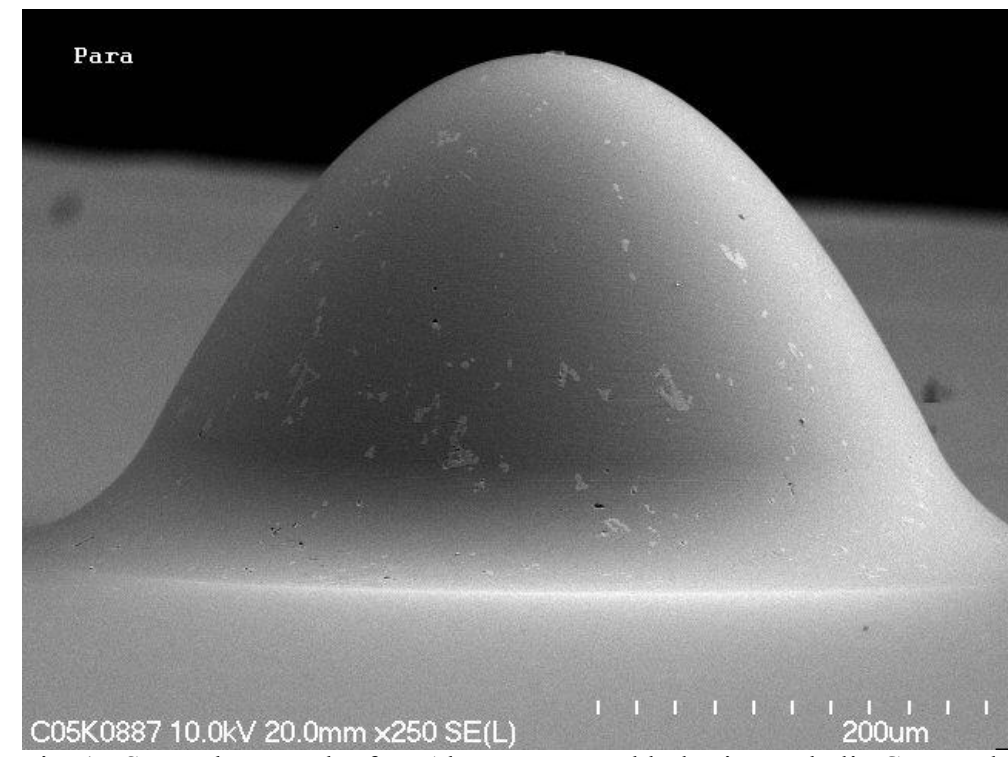

Fig. 1. SEM Photograph of an $\mathrm{Al}$ master to mold plastic parabolic CRLs. The shape of the master is $y=\frac{R^{2}}{100 \mu m}$ where $\mathrm{y}$ is the vertical dimension and $\mathrm{R}$ is the horizontal dimension. The radius of curvature of the lens is $50 \mu m$. is: ${ }^{5}$

$$
2 r_{a}=4\left(\frac{\delta f}{\mu}\right)^{\frac{1}{2}}
$$

This implies that the aperture, and consequently other important lens parameters, such as resolution and depth of field of a CRL, are determined by the required focal length and the choice of material of which the lenses are made. In the experiment two parabolic CRLs were used: a 92-element lens and a 155-element lens. The 92-element lens has a $15.3 \mathrm{~cm}$ focal length and the absorption aperture was $133 \mu \mathrm{m}$. Thus the numerical aperture (NA) was $4.3 \times 10^{-4}$. The 155 -element lens has a $9.3 \mathrm{~cm}$ focal length; the absorption aperture was $104 \mu \mathrm{m}$, and the NA was $5.6 \times 10^{-4}$.

The microscope consists of four main components: an x-ray source (a microspot x-ray tube or an area x-ray tube), multilayer optics, a CRL (parabolic coin CRL or a spherical bubble CRL), and an x-ray detector. The positioning of the various components is shown in Fig. 2. The two x-ray sources that were used are: (a). a microspot $\mathrm{x}$-ray tube with a $\mathrm{Cu}$ anode, Apogee XTG5011 from Oxford Instruments. The nominal focal spot size is $35 \mu \mathrm{m}$, and (b) a Brand X-ray $\mathrm{Cu}$ anode $\mathrm{x}$-ray tube with an apparent source size of $5 \times 5 \mathrm{~mm}^{2}$. The electron-tube voltage was set to $25 \mathrm{keV}$ and a current of $1 \mathrm{~mA}$, resulting in a standard bremsstrahlung and 8-keV-characteristic-line spectra from the microspot tube with no filtering. The Brand X-ray area tube was operated at $30 \mathrm{kV}$ and $15 \mathrm{~mA}$. The detector consists of a Peltier-cooled, 1300 X 1030 format $(6.7 \mu \mathrm{m}$ x $6.7 \mu \mathrm{m}$ pixels) CCD with a fiber-optic-coupled scintillator (Photonic Sciences Ltd., "X-ray 
Fast Digital Imager"). The multilayer optics is a Confocal Max-Flux Optics, CMF-12-38Cu6, from Osmic Inc. A multilayer optic is an x-ray mirror that reflects only one wavelength (energy). Thus, a multilayer provides an ideal, monochromatic spectrum for imaging with a CRL. However, it is not sufficient that the radiation be monochromatic; the $\mathrm{x}$-rays must also pass through the full field of view on the sample and converge toward the aperture of the CRL. The condition is met by focusing optics developed for x-ray diffraction studies. The optic was designed to deliver the

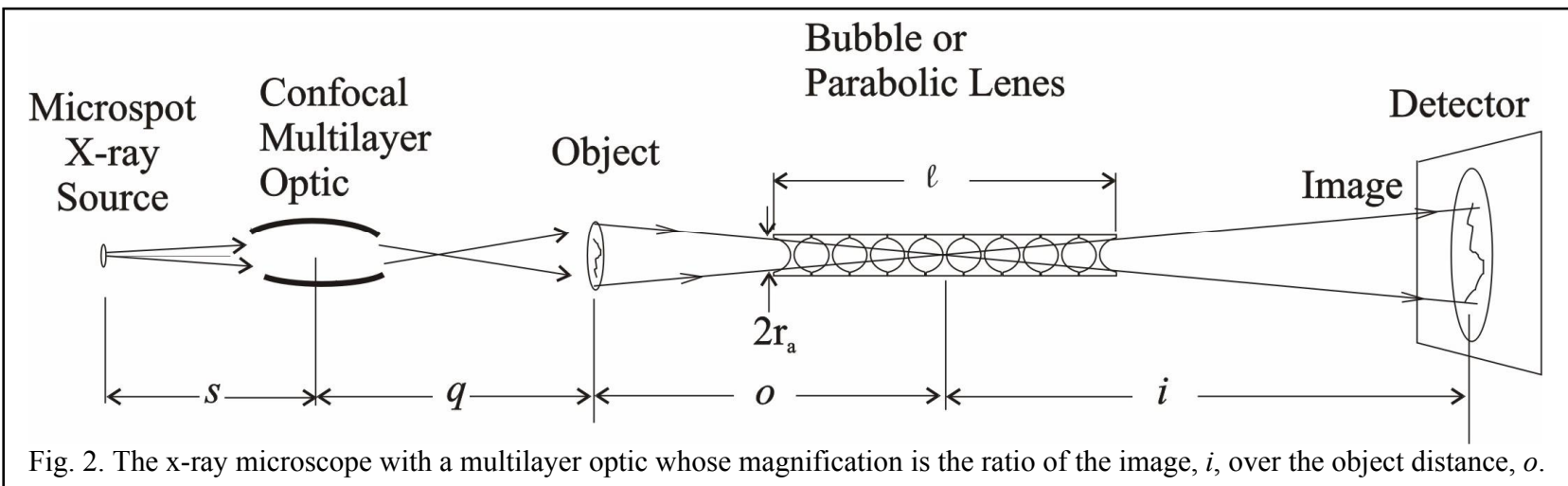

maximum possible $\mathrm{x}$-ray flux to a $200 \mu \mathrm{m}$ spot with minimum divergence. The optic has an approximately $1 \mathrm{~mm} \mathrm{x} 1$ $\mathrm{mm}$ output aperture and focuses to a spot $38 \mathrm{~cm}$ away from the optic. Importantly, the local divergence at each point of the output is approximately $1 \mathrm{mrad}$, which is sufficiently to fill the aperture of the CRL for each point on the object. If the divergence were too small, not enough $\mathrm{x}$-rays would be collected by the lens to form an image.

\section{IMAGING RESULTS}

Images of objects were obtained using either a microspot or an area x-ray tube source. The detector noise was subtracted out, and the source background was compensated. Thus, images were taken with the object (raw image) and without the object (source image). Also an image without source was taken to measure the detector noise (detector noise). The processed image of the object was obtained from (raw image - detector noise) / (source image - detector noise). The process would eliminate any source non-uniformity and inherent detector noise in our imaging system. The images taken of grids are presented in Fig. 3. These images give a strong qualitative presentation of the improved resolution of the imaging system. Fig. 3a was taken with a bubble lens and an area $\mathrm{x}$-ray tube, Fig. $3 \mathrm{~b}$ was with a bubble lens and a microspot tube with the multilayer optics, and Fig. 3c is with a 92-element parabolic lens and a microspot tube and the multilayer optics. Whereas $6-\mu \mathrm{m}$ wires were just resolved in the original system, with improvements to the

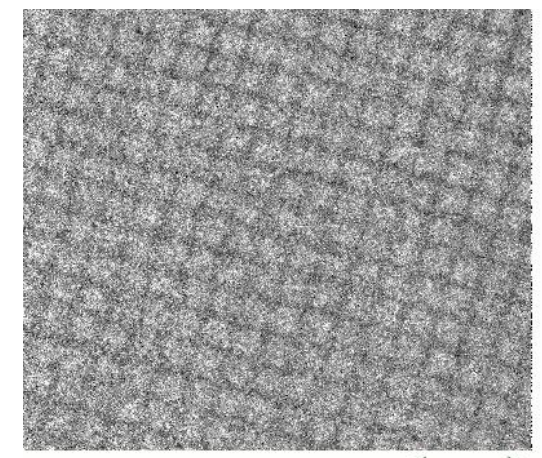

(a)

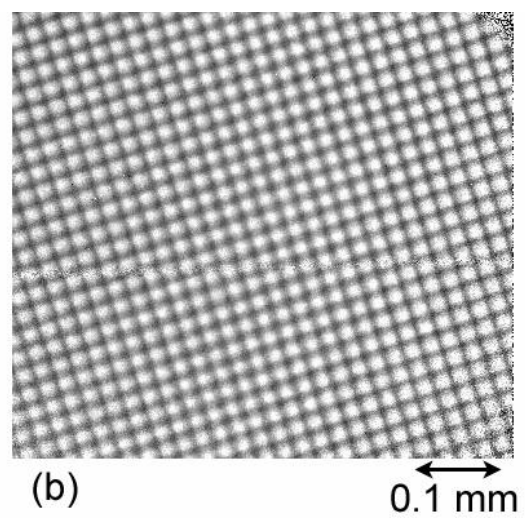

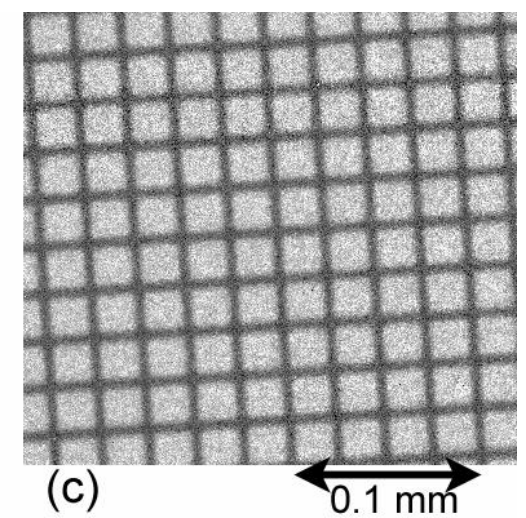

Fig. 3. Images of a 1000 mesh grid $(6 \mu \mathrm{m}$ wires separated by $19 \mu \mathrm{m})$ taken with: a) bubble lens and unfiltered area source at $4 \mathrm{X}$ magnification, at $250 \mathrm{~W}$ for $30 \mathrm{~min}$; (b) parabolic lens and multilayer optic at $4 \mathrm{X}$ magnification, at $25 \mathrm{~W}$ for $10 \mathrm{~min}$; (c) parabolic lens and multilayer optic at $10 \mathrm{X}$ magnification, at $25 \mathrm{~W}$ for $10 \mathrm{~min}$. 
source and CRL, 6- $\mu \mathrm{m}$ wires are clearly resolved and with much greater contrast. Indeed, even at 2.5 times greater magnification the contrast improved over the previous system.

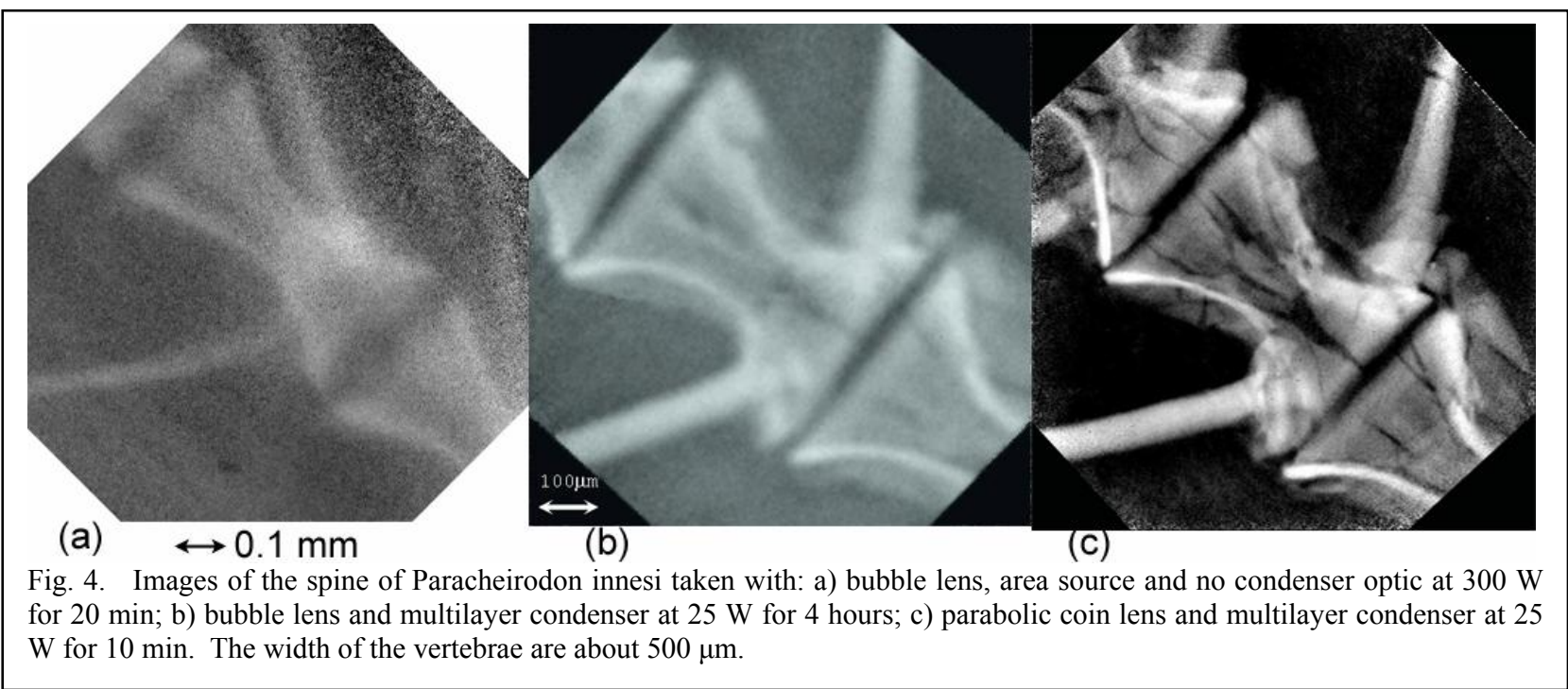

One imaging task that we repeated was looking at the cartilage of a Paracheirodon innesi, or neon tetra aquarium fish. In Fig. 4a, we presented an image of a spine of this fish with a bubble lens and an area $\mathrm{x}$-ray tube. ${ }^{1}$ Fig. $4 \mathrm{~b}$ is taken with a bubble lens and a microspot tube with the multilayer optics, and Fig $4 \mathrm{c}$ is with the 92-element parabolic lens and the multilayer optics. Given our improved resolution, we zoomed in to image individual vertebrae as shown in Fig. 4b and $4 \mathrm{c}$. These images show clear improvement in the image quality. Note that the cracks seen in the parabolic lens image Fig. 4c may not have been in the sample at the time previous images were taken as sample had dried between experiments. We also imaged a Caribbean Grass Sponge to look at a lower contrast object with fine features and to look at the effects of contrast agents. Fig. 5a, b, and c were taken with the 92-element parabolic lens and a microspot tube with the multilayer optics. These were not tightly controlled medical experiments, but rather a preliminary qualitative examination. We looked at three conditions: a natural sponge, a sponge with $1 \%$ iodine solution, and a sponge with Fenestra VC vascular contrast agent, which was provided to us a trial by its producer, Alerion Biomedical. ${ }^{7}$ Both the iodine and Fenestra $\mathrm{VC}$ were added by soaking and then wringing the sponge to remove the liquid, leaving only a residual quantity in the sponge. While there is sufficient contrast to see the sponge without a contrast agent, the agents

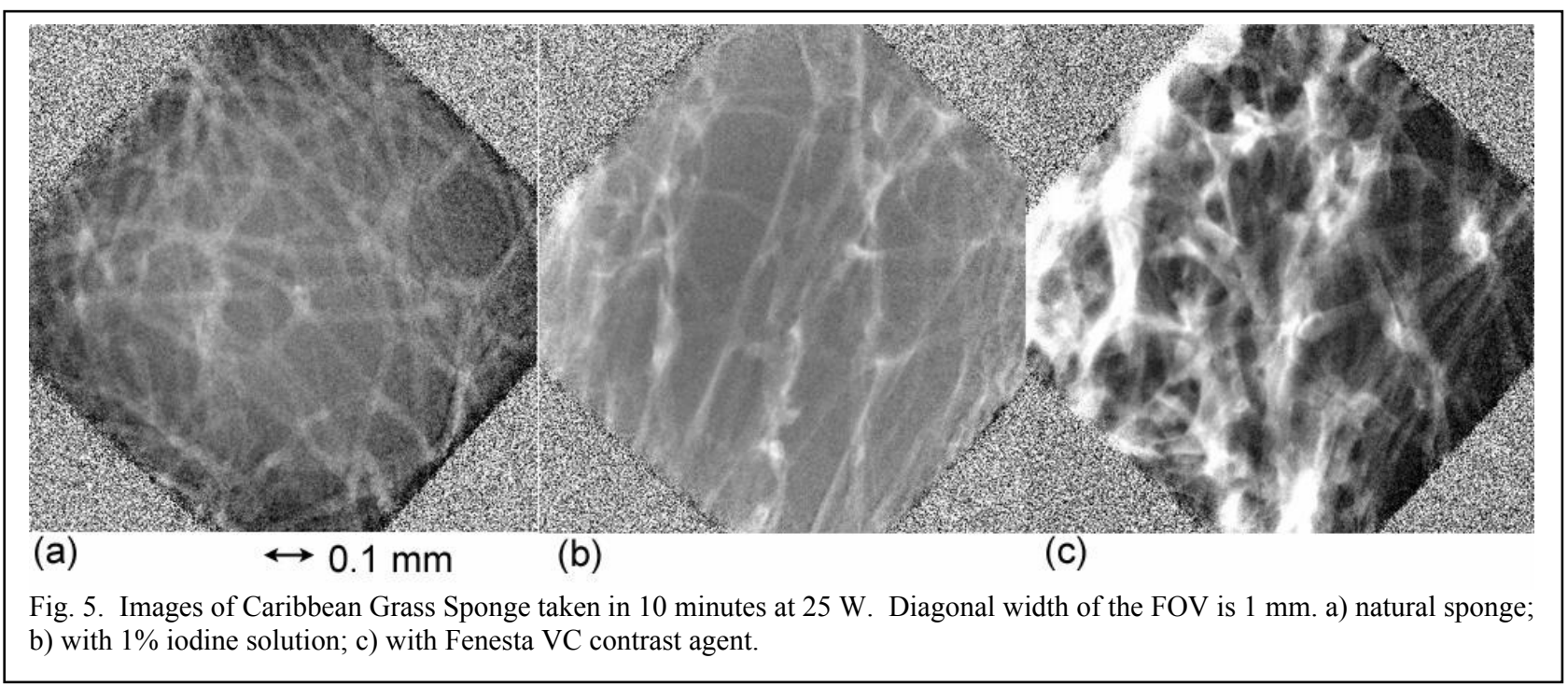


do increase the available signal. Interestingly, the Fenestra VC appears to thicken the strands of the sponge, which may be due to size of the molecules used in the agent, though further study would be needed to confirm this.

The resolution was estimated by drawing a line profile across the image of $25 \mu \mathrm{m}$ thick gold foil, taken with the 155-element parabolic lens at the magnification at 18.4. We took 5 lineprofiles from the image and add them up. The line-profile of the measured knife edge response is given in Fig. 6. The resolution of one of the edges is seen to be $1.1 \mu \mathrm{m}$ (distance between the changes in contrast of $20 \%$ to $80 \%$ ). This resolution is far above the diffractionlimited resolution of $0.61 \lambda / N A=169$ $\mathrm{nm}$. The minimum resolution due to the detector pixel size can be calculated at $\mathrm{M}$ $=18.4$ as $6.7 \mu \mathrm{m} \mathrm{x} 2 / 18.4=0.73 \mu \mathrm{m}$.

Here we assume at least 2 pixels are needed to resolve an object. The use of parabolic lenses and some form of filtering of the source are essential to improving image resolution. Earlier we reported $6-\mu \mathrm{m}$ resolution with a spherical bubble lens and with an area x-ray tube source. ${ }^{1}$ Resolutions better than $0.3 \mu \mathrm{m}$ have been achieved by others using highquality parabolic CRLs of $\mathrm{Al}$ and a synchrotron source. ${ }^{3}$

\section{PORTABLE TOMOGRAPHIC SYSTEM}

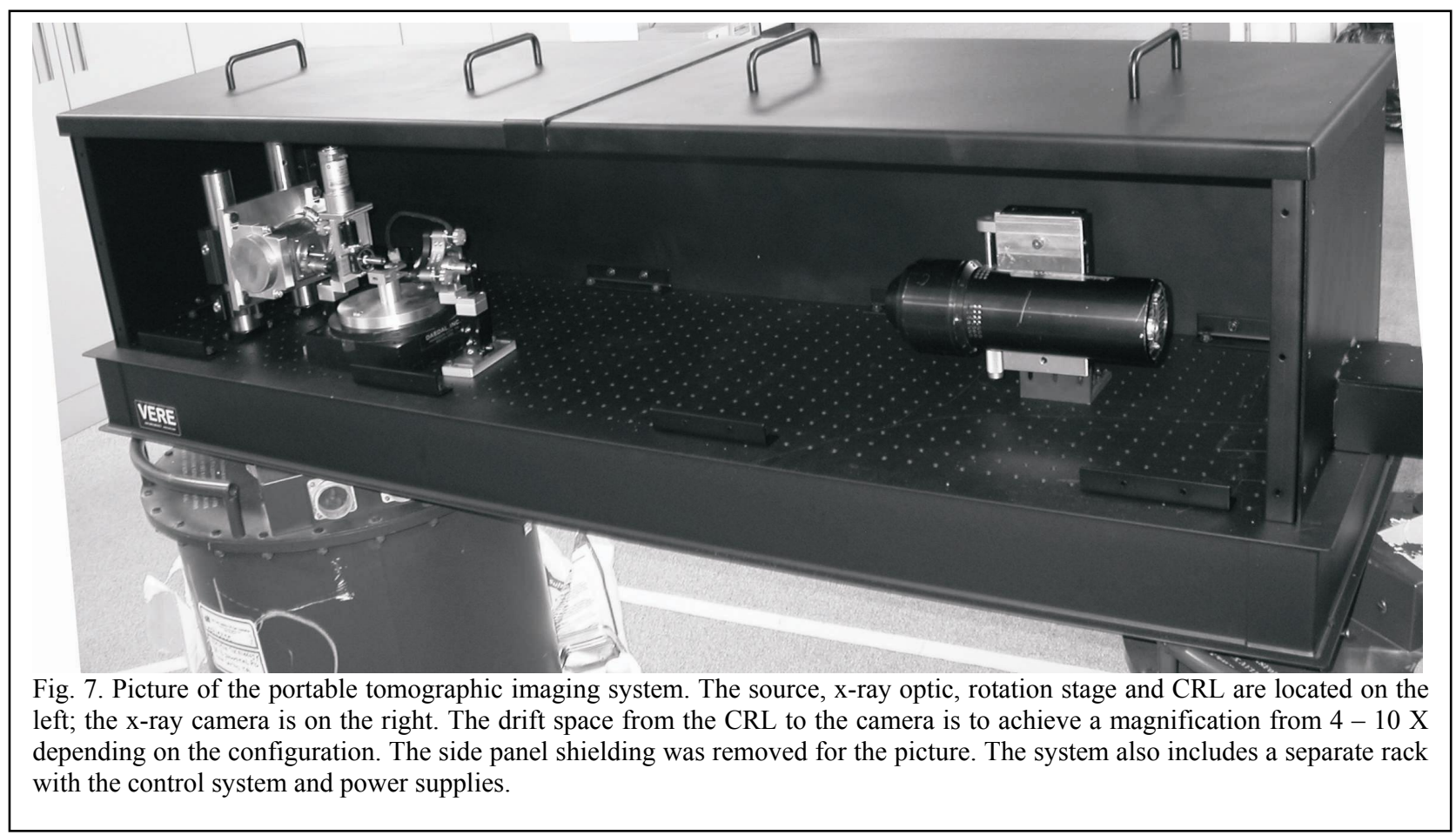


The imaging system was moved to a $46 \mathrm{~cm} \times 152 \mathrm{~cm}$ optical platform to create a portable system. In addition, a rotation stage was added to allow for tomographic imaging. The resulting system has the same functional layout as in Fig. 2 except that the object is now mounted on a rotation stage. In later experiments the multilayer optic was replaced by a polycapillary optic to save space. The polycapillary also delivered about twice as much flux at the imaging plane due to its greater acceptance angle. However, the illumination pattern from the polycapillary is not uniform, requiring more data processing than with the multilayer.

\section{TOMOGRAPHIC IMAGES}

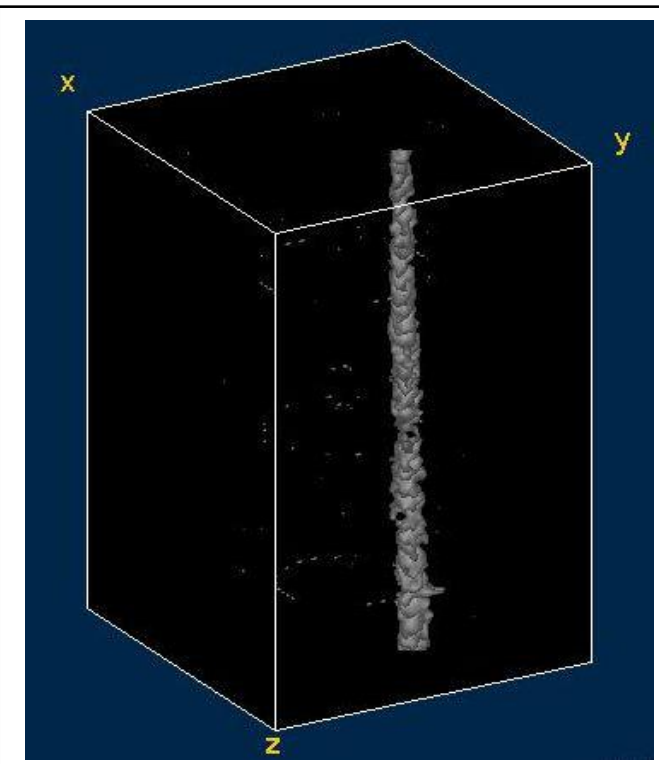

Fig. 8. Tomographic reconstruction of $10-\mu \mathrm{m}$ diameter gold wire.

The first object we imaged was a $10-\mu \mathrm{m}$ diameter gold wire. This object was chosen to test the resolution and help calibrate the system. With a wire, it is easy to measure the position and tilt of the axis of rotation. This information is necessary to properly reconstruct objects. Scans were taken at 60 evenly spaced angles. Each scan image took one minute, for a total imaging time of 60 minutes. This exposure time can be greatly improved by using a more powerful x-ray source/condenser optic combination. The resulting tomgraphic reconstruction of the wire is shown in Fig. 8. The surface of the wire is uneven due to a combination of image noise and the finite effective pixel size of the camera $(1.7 \mu \mathrm{m}=6.7$ $\mu \mathrm{m} / 4 \mathrm{X}$ magnification).

Work is proceeding on acquiring tomographic data of other objects. In addition, the resolution of the system will be improved by changing to a higher resolution scintillator and a more precisely parabolic CRL. A higher accuracy rotation stage is also required. The throughput will be improved by using a more efficiently coupled, higher power source and a more sensitive x-ray camera.

\section{ACKNOWLEDGEMENTS}

This work was supported by the United States Department of Health and Human Services under the Small Business Innovative Research Program (Grant \# 2 R44 RR021242-02), the United States National Science Foundation under the Small Business Innovative Research Program (Grant \#DMI-0450518), and by the Byelorussian Foundation of Fundamental Investigations (Grant \# F03MC-005).

\section{REFERENCES}

1. M. A. Piestrup, C. K. Gary, H. Park, J. L. Harris, J. T. Cremer, R. H. Pantell, Y. I. Dudchik, N. N. Kolchevsky, and F. F. Komarov, Appl. Phys. Lett. 86, 131104 (2005).

2. Y. I. Dudchik, N. N. Kolchevsky, F. F. Komarov, M. A. Piestrup, J. T. Cremer, C. K. Gary, H. Park, and A. M. Khounsary, Rev. Sci. Instrum. 75, 4651 (2004)..

3. B. Lengeler, C. G. Schroer, M. Richwin, J. Tümmeler, M. Drakopolulos, A. Snigirev and I. Snigireva, Appl. Phys. Lett. 74, 3924 (1999).

4. Y. Kohmura, K. Okada, A. Takeuchi, H. Takano, Y. Suzuki, T. Ishikawa, T. Ohigashi, and H. Yokosuka, Nucl. Instrum. Meth. A467-468, 881 (2001).

5. A. Snigirev, V. Kohn, I. Snigireva and B. Lengeler, Nature (London) 384, 49 (1996).

6. R. H. Pantell, J. Feinstein, H. R. Beguristain, M. A. Piestrup, C. K. Gary, and J. T. Cremer, Appl. Opt. 42, 719 (2003).

7. Alerion Biomedical Inc., San Diego, CA 92121. Tel. 858-558-2191 